\title{
Quit When You Can: Efficient Evaluation of Ensembles by Optimized Ordering
}

\author{
SERENA WANG and MAYA GUPTA, Google Research, Mountain View, CA, USA \\ SEUNGIL YOU, Kakao Mobility, Jeju City, Republic of Korea
}

\begin{abstract}
Given a classifier ensemble and a dataset, many examples may be confidently and accurately classified after only a subset of the base models in the ensemble is evaluated. Dynamically deciding to classify early can reduce both mean latency and CPU without harming the accuracy of the original ensemble. To achieve such gains, we propose jointly optimizing the evaluation order of the base models and early-stopping thresholds Our proposed objective is a combinatorial optimization problem, but we provide a greedy algorithm that achieves a 4-approximation of the optimal solution under certain assumptions, which is also the best achievable polynomial-time approximation bound. Experiments on benchmark and real-world problems show that the proposed Quit When You Can (QWYC) algorithm can speed up average evaluation time by 1.8-2.7 times on even jointly trained ensembles, which are more difficult to speed up than independently or sequentially trained ensembles. QWYC's joint optimization of ordering and thresholds also performed better in experiments than previous fixed orderings, including gradient boosted trees' ordering.
\end{abstract}

\section{CCS Concepts: • Computing methodologies $\rightarrow$ Ensemble methods; Cost-sensitive learning;}

Additional Key Words and Phrases: Efficient ensemble evaluation, ensemble learning, combinatorial optimization, gradient boosting

\section{ACM Reference format:}

Serena Wang, Maya Gupta, and Seungil You. 2021. Quit When You Can: Efficient Evaluation of Ensembles by Optimized Ordering. f. Emerg. Technol. Comput. Syst. 17, 4, Article 55 (July 2021), 20 pages.

https://doi.org/10.1145/3451209

\section{INTRODUCTION}

We consider the problem of efficiently evaluating a binary classifier that can be expressed as an ensemble of $T$ base models:

$$
f(x)=\sum_{t=1}^{T} f_{t}(x),
$$

where each base model $f_{t}(x) \in \mathbb{R}$ contributes a score. The ensemble may have been trained:

- Independently: Each $f_{t}$ is trained individually, e.g., random forests [4].

- Sequentially: The $t$ th base model $f_{t}$ is trained taking into account base models $f_{1}, f_{2}, \ldots$, $f_{t-1}$, e.g., boosted trees [11].

Authors' addresses: S. Wang and M. Gupta, Google Research, 1600 Amphitheatre Parkway Mountain View, CA, 94043, USA; emails: \{serenawang, mayagupta\}@google.com; S. You, Kakao Mobility, Republic of Korea, 242 Cheomdan-ro Jeju-Si, Jeju-do, 63309, Republic of Korea; email: sean.you@kakaomobility.com.

\section{(i)}

This work is licensed under a Creative Commons Attribution International 4.0 License.

(C) 2021 Copyright held by the owner/author(s).

1550-4832/2021/07-ART55 \$15.00

https://doi.org/10.1145/3451209

ACM Journal on Emerging Technologies in Computing Systems, Vol. 17, No. 4, Article 55. Pub. date: July 2021. 
- Jointly: All base models $\left\{f_{t}\right\}$ are trained together, e.g., generalized additive models [14] or an ensemble of lattices [6].

Ensemble pruning methods can be categorized as static or dynamic. In traditional static ensemble pruning, a subset of base models is selected from the full ensemble, and that same subensemble is used to evaluate all examples $([18,19,24,32,42]$, and many others). In contrast, in dynamic ensemble pruning, the subensemble selected depends on the example evaluated, the idea being that different base models may be better suited for evaluating different examples [38]. While most studies compare within their respective static and dynamic ensemble pruning categories, surveys have demonstrated that dynamic ensemble pruning can achieve better speedups than static [33, 37]. Dynamic pruning can also be complementary to the traditional static ensemble pruning approach, as the dynamic approach can act on a pre-pruned ensemble to further reduce computation requirements per example.

Many dynamic ensemble pruning strategies involve ordering the base models by some heuristic (such as accuracy) and selecting subsets of base models as prioritized by the ordering. Woods et al. [37] introduced the idea of first clustering the examples, then ordering the base models by accuracy within each cluster, then choosing the top $N$ base models within each cluster for some fixed $N$. Santana et al. [26] showed that ordering base models by both accuracy and diversity can actually produce more accurate subensembles at test time. Santos et al. [27] proposed a slightly different heuristic of choosing the most confident base models for each example. Fan et al. [9] suggested ordering by total benefits of the base models, where total benefits may include some combination of accuracy and the classifier's objective. Fan et al. [9] also apply a more complex early stopping mechanism where the size of the subensemble also depends on the difficulty of each example. The ultimate goal of the ordering and early stopping mechanism is to achieve faster evaluation time without sacrificing too much performance compared to the full ensemble.

Like Fan et al. [9], we leverage a key idea in dynamic pruning that examples that are easy to classify can be confidently and accurately classified after evaluating only a few base models, but hard examples may require evaluating more base models. This adapts the computation to the difficulty of the classification problem, reducing average latency and computational resources (e.g. $\mathrm{CPU}$ ) needed to classify. Specifically, we apply the following simple early stopping mechanism for a given example $x$ : after evaluating the $r$ th ordered base model, if the accumulated sum $\sum_{t=1}^{r} f_{t}(x)$ is above the $r$ th early positive threshold $\epsilon_{r}^{+}$, then the example is classified as the positive class, and the evaluation is stopped early (and vice-versa if the sum is below the $r$ th negative threshold $\epsilon_{r}^{-}$). If the running sum does not exceed an early stopping threshold, one continues on to evaluate the $r+1$ th base model.

The main challenge to this set-up is to select an a priori ordering of the base models, and corresponding early stopping thresholds. While ordering by total benefits, diversity, and confidence have been shown to work better than ordering by accuracy alone for achieving faster evaluation time, we aim to bypass these heuristics entirely by explicitly optimizing the order to improve the evaluation time objective, with a constraint that the decisions do not change with respect to the original ensemble. That is, we propose a combinatorial optimization problem that minimizes the average number of base models that need to be evaluated to achieve the same classification decision as the full classifier. We simultaneously optimize the $2 T$ corresponding early decision threshold vectors $\left\{\epsilon^{+}, \epsilon^{-}\right\}$.

While finding a globally optimal solution for this combinatorial optimization problem is NPhard, we propose a greedy algorithm we call Quit When You Can (QWYC). Under certain assumptions, we show that QWYC achieves a 4-approximation of the optimal solution, which is also the best approximation bound that can be achieved in polynomial time. 
Experiments on both real-world and benchmark problems show that QWYC can speed up computation time by $1.8-2.7 \times$ even on highly optimized jointly trained ensembles, while only changing a few classification decisions on held-out examples from the full ensemble's evaluation. QWYC's joint optimization of ordering and early stopping mechanism also performed better in experiments than various fixed heuristic orderings, including the natural ordering produced during training of gradient boosted trees (Section 5). This performance held even for datasets with strongly imbalanced class priors (Experiments 3 and 5).

Overall, the main contribution of this work is demonstrating both the value and practicality of jointly optimizing the ordering and early stopping mechanism for dynamic ensemble pruning.

\section{RELATED WORK}

A closely related dynamic ensemble pruning strategy was proposed by Fan et al. [9]. Their approach orders the base models based on their individual utility. For example, they order from the base model that has the highest accuracy when used by itself to the base model that has the lowest accuracy when used by itself. They propose an early stopping mechanism where the threshold to declare an early positive or negative class depends on a probabilistic modeling of the running score. A more detailed explanation and implementation is given in Section 5.4. In contrast, we take a discriminative approach: we propose to jointly optimize the ordering with the early stopping thresholds to minimize the expected evaluation cost, constrained to producing the same classification decisions as the full classifier within a given error margin.

A similar approach of optimizing the ordering of base models was proposed by Sun and Zhou [31], but their work relies on the assumption that each base model can only output one of two possible discriminant values: $f_{t}(x) \in\left\{\alpha_{t}, \beta_{t}\right\}$ for $\alpha_{t} \leq \beta_{t} \in \mathbb{R}$. We focus on the more general problem where we do not assume $f_{t}(x)$ is discrete or even bounded (e.g., ensembles of neural networks [42], GAMs [14], lattices [6], and various combinations thereof [22]). Although we discriminatively learn early stopping thresholds, Sun and Zhou [31] build on an early stopping mechanism where the evaluation stops if the remaining base models cannot alter the final decision, relying on the assumption that $f_{t}(x)$ is binary and bounded across all inputs $x$ (e.g., FastExit and variants [16, 29, 41]). Like Sun and Zhou [31], we empirically show that optimizing the ordering can produce a better tradeoff in evaluation time and test error than other heuristic orderings. We further provide a theoretical approximation bound on the optimality of our polynomial-time ordering algorithm.

Another common approach in dynamic ensemble pruning involves using clustering to match examples to their most accurate subensembles. These methods require the user to specify an ordering criteria for each cluster [37]. Some work has been done on improving the ordering of base models for each cluster by selecting for both accuracy and diversity instead of just accuracy [26, 38]. Our method is thus complementary to these clustering approaches - for examples in each cluster, QWYC can choose an ordering that directly reduces evaluation time rather than relying on the user to specify selection heuristics.

A special case of the efficient ensemble evaluation problem is when the base models are assumed to be independently trained and randomly ordered, and each base model only contributes a vote of yes or no for each class, rather than a score. Then the incomplete votes can be modeled as draws from a multinomial distribution [15, 28] and the class priors can be incorporated [30]. Other researchers have shown similar results using a Gaussian approximation [1]. In those papers, only intermediate thresholds for stopping early are chosen; the base models are not re-ordered as part of the speed-up.

Rather than define explicit thresholds, Benbouzid et al. [2] propose a more complex early stopping mechanism by building a Markov Decision Process where, at each node, one can decide to use 
or skip each base model. They take the ordering as given and thus do not explore the relationship between the ordering and early stopping mechanism.

Many of these approaches can be interpreted as a form of stacking [36], where the base model outputs are sent to a meta-learner. Cruz et al. [7] explicitly trains a meta-learner to reorder the base models by "competence," Kim et al. [16] train a decision tree as a meta-learner when each base model outputs a binary decision. Our proposed QWYC algorithm can be viewed as a lightweight stacking decision tree with one base model per decision node.

A related but different problem is trying to adaptively minimize the set of features needed to evaluate each $x$, either to reduce costs or time associated with obtaining those features $[21,23,35]$ In this article, we do not explicitly model feature computation at all. We depend only on the cost of evaluating each base model and treat each base model evaluation cost as independent of the cost of evaluating other base models. One could model feature evaluation costs via the base model evaluation cost, but if two base models use the same expensive feature, then our model would not take into account that the second use of the feature was essentially free.

A more general version of the problem than what we tackle here is that instead of using the same optimized ordering of the base models for all examples, one could adaptively choose which base model to evaluate next $[13,39]$. Such instance-adaptive orderings could potentially offer even greater gains if some of the base models are relatively expensive to evaluate. However, these orderings would require substantially greater system complexity in practice.

Others have focused on how to design and train a cascade of base models to enable significant early rejections [3, 17, 25, 34, 40]. For example, Viola and Jones [34] produced a seminal real-time face recognition systems by hand-designing a simple-to-complex cascade of classifiers that could perform early rejection. In this article, we take the ensemble as given, and propose a method for speeding up a given already-trained ensemble.

Last, another strategy for fast evaluation of ensembles is to evaluate the base models in parallel. However, parallelization does not reduce the mean computational resources needed to classify, which is one of the goals of this work. Moreover, parallelized evaluation is a complementary strategy: one can parallelize sub-ensembles of base models such that each $f_{t}$ represents one subensemble. In practice, if one can evaluate batches of samples at a time, we have also found it less complex in terms of software engineering and more efficient to simply parallelize over samples rather than parallelizing over base models.

\section{OPTIMIZATION PROBLEM}

We take as given a linearly separable model $f(x): x \in \mathbb{R}^{D} \rightarrow \mathbb{R}$ such that $f(x)=\sum_{t=1}^{T} f_{t}(x)$, an expected time (or other cost) $c_{t} \in \mathbb{R}^{+}$to evaluate the $t$ th base model $f_{t}(x)$ for $t=1, \ldots, T$, and a decision threshold $\beta \in \mathbb{R}$ for classifying $f(x)$.

We propose producing a fast classifier by optimizing the ordering of base models over the set of all permutations $\Pi$ that map $\{1,2, \ldots, T\} \rightarrow\{1,2, \ldots, T\}$, and over the positive and negative threshold vectors $\epsilon^{+}, \epsilon^{-} \in \mathbb{R}^{T}$ to minimize the expected evaluation cost of a random sample $X \in$ $\mathbb{R}^{D}$ drawn from a data distribution $\mathbb{P}_{X}$, with a constraint specified as a percent $\alpha$ of samples that may be classified differently by the fast classifier compared to the full classifier $f(x)$ :

$$
\begin{array}{ll}
\underset{\epsilon^{-}, \epsilon^{+} \in \mathbb{R}^{T}, \pi \in \Pi}{\operatorname{minimize}} & \mathbb{E}_{X}\left[\sum_{t=1}^{T} c_{\pi(t)} I_{X, t, \pi, \epsilon^{+}, \epsilon^{-}}\right] \\
\text {subject to } & \mathbb{E}_{X}\left[Z_{\left.X, t, \pi, \epsilon^{+}, \epsilon^{-}\right] \leq \alpha,}\right. \\
& \epsilon_{t}^{-} \leq \epsilon_{t}^{+} \text {for all } t,
\end{array}
$$


where $I_{X, t, \pi, \epsilon^{+}, \epsilon^{-}}$is a Bernoulli random variable that is 1 if $X$ is not classified yet after evaluating $t$ base models in the ordering $\pi$ and with early-stopping decision threshold vectors $\epsilon^{+}$and $\epsilon^{-}$, and is 0 otherwise; and $Z_{X, \pi, \epsilon^{+}, \epsilon^{-}}$is a Bernoulli random variable that is 1 if the fast classification of $X$ differs from the full evaluation classifier decision for $X$, and 0 otherwise.

There are several practical advantages for constraining the fast classifier in terms of differences to the full classifier's decision, rather than constraining it in terms of its error rate. First, this same choice is made in related work (e.g., Parrish et al. [23]). Second, there always exists a feasible solution for all $\alpha \geq 0$. Third, the constraint can be evaluated on unlabeled data, which is often cheaper to obtain than labeled data. Fourth, since labels are not needed, one can optimize the fast classifier on the actual runtime distribution of examples, which may not be IID with the training distribution.

\subsection{Empirical Approximation}

In practice, we do not have direct access to $\mathbb{P}_{X}$ and instead we take as given a set of $n$ examples $\left\{x_{i}\right\}$ assumed to be sampled independently and identically from $\mathbb{P}_{X}$. Let $g_{r}(x, \pi)$ denote the accumulated sum (incomplete score) after $r$ base models ordered as per $\pi: g_{r}(x, \pi)=\sum_{t=1}^{r} f_{\pi(t)}(x)$.

After evaluating the $r$ th base model for $r \in\{1, \ldots, T\}$, an example $x$ belongs to one of three mutually exclusive sets: the predicted positive set $P_{r}:=\left\{x \mid g_{r}(x, \pi)>\epsilon_{r}^{+}\right\}$, the predicted negative set $N_{r}:=\left\{x \mid g_{r}(x, \pi)<\epsilon_{r}^{-}\right\}$, and the uncertain set $U_{r}:=\left\{x \mid \epsilon_{r}^{-} \leq g_{r}(x, \pi) \leq \epsilon_{r}^{+}\right\}$. Note $U_{0}=\mathbb{R}^{D}$, $P_{0}=\emptyset, N_{0}=\emptyset$. If $x \in P_{r}$ or $x \in N_{r}$, then the fast classifier classifies $x$ as the positive or negative class, respectively, and we terminate the evaluation. Otherwise, $x \in U_{r}$, the classification decision is not yet uncertain, and we continue on to evaluate the $\pi(r+1)$ th base model. Let $C_{r}:=\bigcap_{t=0}^{r} U_{t}$ denote the set of samples that remain unclassified after evaluating the $r$ th base model.

For the full classifier, we define two mutually exclusive sets: the negative decisions $N_{\text {full }}:=$ $\{x \mid f(x)<\beta\}$, and the positive decisions $P_{\text {full }}:=\{x \mid f(x) \geq \beta\}$.

To produce a fast classifier, we approximate objective (2) with the empirical analogue, using $\mathbf{1}[\cdot]$ to denote the standard indicator function:

$$
\begin{aligned}
\underset{\epsilon^{+}, \epsilon^{-} \in \mathbb{R}^{T}, \pi \in \Pi}{\operatorname{minimize}} & \frac{1}{N} \sum_{t=1}^{T} \sum_{i=1}^{N} c_{\pi(t)} \mathbf{1}\left[x_{i} \in C_{t-1}\right] \\
\text { subject to } & h_{T}\left(\epsilon^{+}, \epsilon^{-}, \pi\right) \leq \alpha \\
& \epsilon_{t}^{-} \leq \epsilon_{t}^{+} \text {for all } t,
\end{aligned}
$$

where $h_{T}\left(\epsilon^{+}, \epsilon^{-}, \pi\right)$ represents the fraction of classification differences between the fast classifier and the full classifier:

$$
h_{T}\left(\epsilon^{+}, \epsilon^{-}, \pi\right)=\frac{1}{N} \sum_{i=1}^{N}\left(1\left[x_{i} \in \bigcup_{t=1}^{T}\left\{C_{t-1} \cap P_{t} \cap N_{\text {full }}\right\}\right]+1\left[x_{i} \in \bigcup_{t=1}^{T}\left\{C_{t-1} \cap N_{t} \cap P_{\text {full }}\right\}\right]\right)
$$

In Section 4, we give an algorithm to approximately optimize (3).

Representative Dataset. Certainly, the value of the solution to (3) will depend on how representative the examples $\left\{x_{i}\right\}$ are of the examples that will be seen in actual usage of the model. Because optimizing (3) does not require labels for the examples, it can be optimized on unlabeled examples sampled from the runtime distribution.

Filtering Candidates. An important use case in practice that calls for fast classification is when one only wishes to reject examples quickly. For example, when recommending products to a user from a database of millions of products, one hopes to quickly reject most products, but the most promising products need to be fully scored, so that a ranked list of relevant products can be shown 
to the user. For such cases, objective (3) can be simplified to only finding early-rejection thresholds $\left\{\epsilon_{t}^{-}\right\}$, as any positively classified samples will need the full score and must thus be fully evaluated.

\section{QUIT WHEN YOU CAN ALGORITHM}

Since (3) is a combinatorial optimization over $\pi$, coupled with a $2 T$-dimensional optimization over the real-valued threshold vectors $\epsilon^{-}$and $\epsilon^{+}$, we have little hope of finding a global optimum except for small $T$. In the next section, we propose a greedy optimization of (3) for both the ordering of the base models and the thresholds, which we refer to as QWYC. Then in Section 4.2 we give some theoretical results for QWYC.

\subsection{QWYC Algorithm}

Our proposed QWYC algorithm is a greedy optimization over the base model ordering. The algorithm first calculates the optimal thresholds to satisfy the constraints of (3) for each of the possible $T$ base models that could be selected as the first base model $\pi(1)$. The base model that minimizes the evaluation time ratio (defined below) is then set as $\pi(1)$ with its corresponding optimized thresholds. The process is then repeated for each of the base models in order, taking into account the previous chosen base models and thresholds. The base models are compared in terms of their evaluation time ratio $J_{t}$, defined as:

$$
J_{t}\left(\epsilon^{+}, \epsilon^{-}, \pi\right)=\frac{c_{\pi(t)} \sum_{i=1}^{N} 1\left[x_{i} \in C_{t-1}\right]}{\sum_{i=1}^{N} 1\left[x_{i} \in\left\{C_{t-1}-C_{t}\right\}\right]},
$$

where the numerator is the total cost to evaluate the $t$ th base model on the set of uncertain examples $C_{t-1}$, but that cost is normalized by the denominator, which is the number of examples that are no longer uncertain after we evaluate the $t$ th base model. Thus, the numerator prefers choosing a base model with low cost to evaluate $c_{\pi(t)}$, but the denominator prefers choosing the base model that will early-classify the most examples. For cases where all base models are equally expensive to classify such that $c_{\pi(t)}$ is a constant, only the denominator matters. Our definition of $J_{t}$ mirrors the cost ratio term in the Pipelined Set Cover problem given by Munagala et al. [20], which we will take advantage of to provide guarantees in the theory section.

Algorithm 1 details this procedure, where we have pulled out the QWYC optimization of the thresholds into a subroutine detailed in Algorithm 2. Optimizing (3) for each $\epsilon_{r}^{-}$can be done using the bisection method [5], since the objective is monotonically decreasing with respect to $\epsilon_{r}^{-}$, and the constraint is monotonically increasing with respect to $\epsilon_{r}^{-}$(and analogous logic holds for each $\epsilon_{r}^{+}$). These are detailed in Algorithms 3 and 4. Assuming that the bisection method runs a limited number of iterations over the dataset and is $O(N)$ where $N$ is the size of the dataset, then the time complexity for this procedure is $O\left(T^{2} N\right)$. Algorithm 2 can be applied to compute early stopping thresholds for any ordering of the base models, and in the experiments we compare different ordering methods combined with the QWYC early stopping thresholds.

\subsection{QWYC Approximation Bound}

Under some assumptions, the problem of finding the optimal ordering and early stopping thresholds reduces to the Pipelined Set Cover problem [20], and the QWYC greedy algorithm therefore achieves a 4-approximation of the optimal evaluation time. Suppose at time $i$ we choose to evaluate base model $f_{t}$, then denote the set of examples we can stop early on as $S_{t}(i)$, assuming we were not allowed to stop early on any examples before time $i$. Intuitively, each $f_{t}$ "covers" a set $S_{t}(i)$, and the goal is to find the order of evaluation of the $f_{t}$ 's to cover all the examples with minimal evaluation cost. 

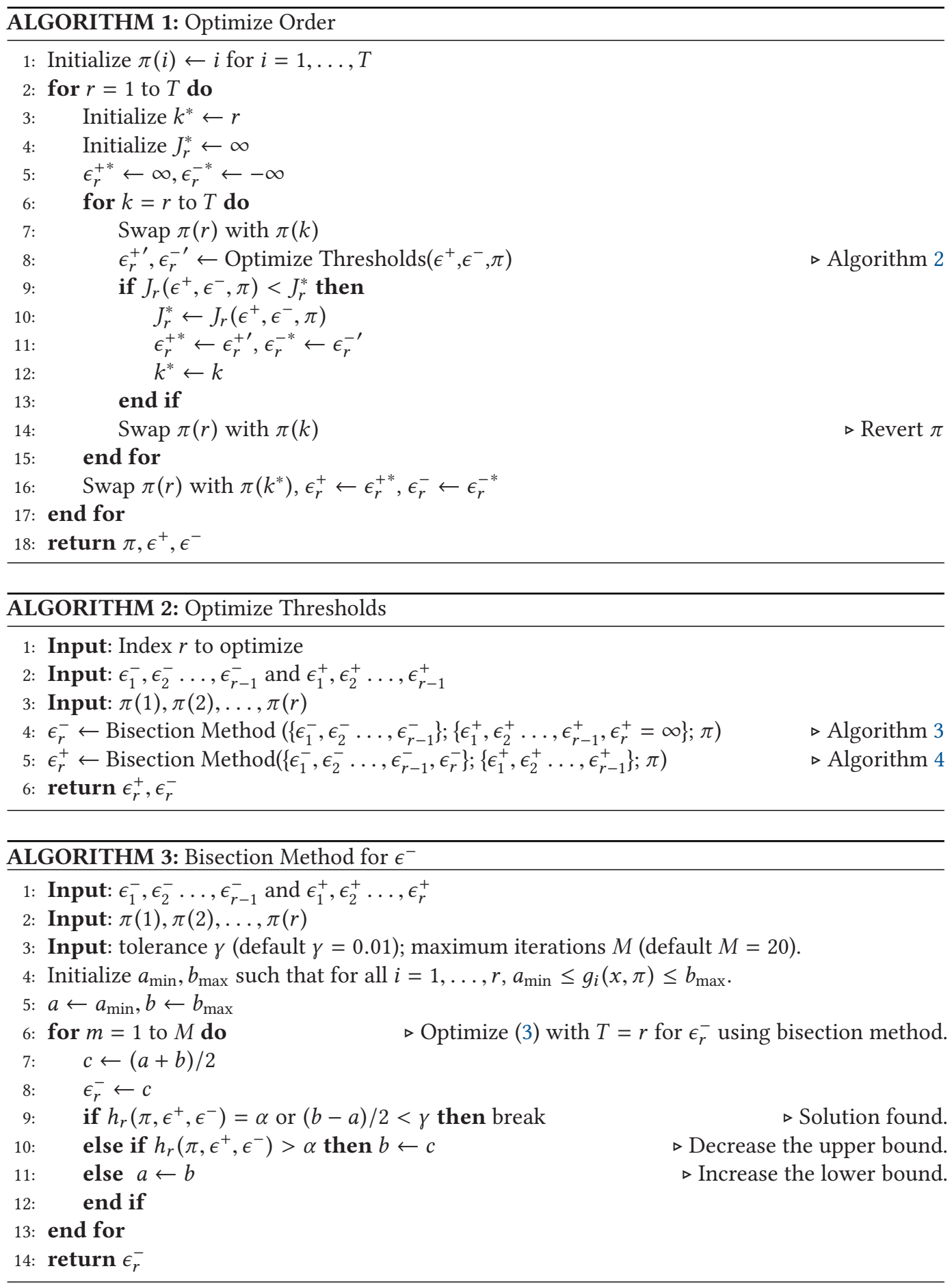

In general, the ordering of base models before $i$ will impact the size of $S_{t}(i)$, but a simple special case called Pipeline describes those problems for which the optimal ordering $S_{t}(i)=S_{t}(1)$. We 


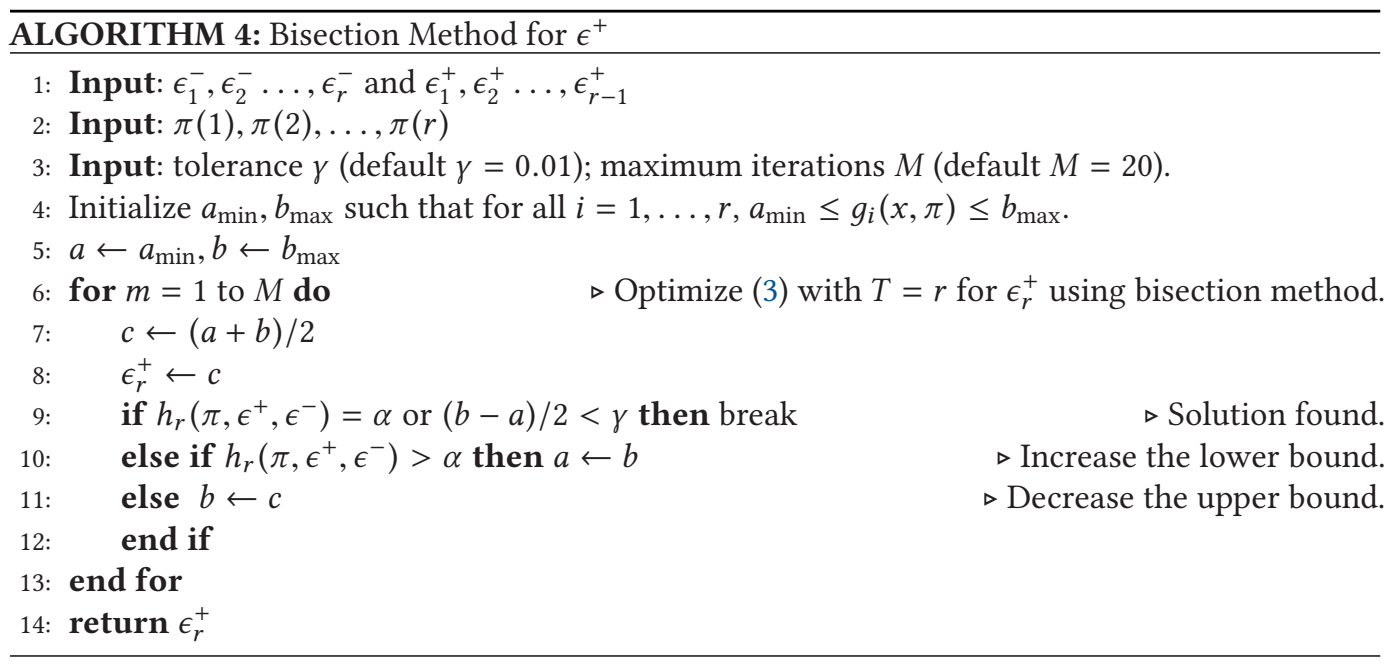

provide a concrete example of a problem from PIPELINE below, and then show QWYC achieves a 4-approximation of the optimal cost for these problems.

Admittedly, Pipeline is a fairly restrictive class, and we do not expect real-world ensembles to satisfy these strict assumptions. However, the following results can help practitioners reason about the kinds of real cases for which the QWYC algorithm is likely to achieve a good approximation: ensembles with diverse base models such that each base model is confident on a disjoint set of examples are more likely to approach this criteria than ensembles with similar base models.

Definition 4.1. Assuming no early stopping was allowed before reaching the $i$ th base model, let $\pi(i)=t$ and define $S_{t}(i)$ to be the maximal set of examples with respect to all choices of $\epsilon_{i}^{+}, \epsilon_{i}^{-}$for which base model $f_{t}$ can make an early negative or early positive classification while satisfying the constraint with $\alpha=0$. Note for $i \geq 1, S_{t}(i) \supseteq S_{t}(1)$. Let $O P T$ be the optimal cost under the conservative restriction that $S_{t}(i)=S_{t}(1)$ for $i \geq 1$. Let $O P T^{*}$ be the true optimal cost. Define Pipeline to be the set of problems where $O P T=O P T^{*}$.

Example Ensemble in Pipeline: Let $\alpha=0$ and let $c_{t}=1$ for all $t$. Suppose there are examples $\left\{e_{1}, \ldots, e_{8}\right\}$ and base models $f_{1}, f_{2}, f_{3}$, and the decision threshold is 0 for the full classifier $\sum_{t=1}^{3} f_{t}$. Let base models $f_{1}, f_{2}, f_{3}$ produce the following scores:

$f_{1}\left(e_{1}\right)=1, f_{1}\left(e_{2}\right)=-1$ and 0 for all others,

$f_{2}\left(e_{3}\right)=1, f_{2}\left(e_{4}\right)=1, f_{2}\left(e_{5}\right)=-1$ and 0 for all others, and

$f_{3}\left(e_{5}\right)=-1, f_{3}\left(e_{6}\right)=1, f_{3}\left(e_{7}\right)=-1, f_{3}\left(e_{8}\right)=-1$ and 0 for all others.

For this problem, $S_{1}(i)=S_{1}(1)=\left\{e_{1}, e_{2}\right\}$ regardless of where $f_{1}$ is evaluated with respect to $f_{2}$ and $f_{3}$. The same is true for $S_{2}(i)$ and $S_{3}(i)$. Thus, the optimal order is $\pi=[3,2,1]$ and the optimal evaluation time cost is

$O P T=O P T^{*}=\frac{1}{8}\left(8 c_{3}+4 c_{2}+2 c_{1}\right)=\frac{7}{4}$.

LEMma 1. For problems in PIPELINE, the QWYC algorithm achieves a 4-approximation of the optimal cost, which is also the best achievable approximation bound.

Proof. The proof is a direct application of prior results studying the Pipelined Set Cover problem by Munagala et al. [20] and Feige et al. [10].

Let $\alpha=0$ and restrict $S_{t}(i)=S_{t}(1)$ regardless of position $i$. Let $O P T$ be the evaluation time cost of the optimal ordering and thresholds under these restrictions. Finding the optimal $\pi, \epsilon^{+}, \epsilon^{-}$under 
Table 1. Datasets and Ensembles Used in Experiments

\begin{tabular}{lcccccc}
\hline Dataset & \# Features & Train & Test & Ensemble type & Ensemble size & Early Stopping \\
\hline UCI Adult & 14 & 32,561 & 16,281 & GBT & 500 & pos. \& neg. \\
UCI Nomao & 8 & 27,572 & 6,893 & GBT & 500 & pos. \& neg. \\
Real-World 1 & 16 & 183,755 & 45,940 & Lattices & 5 & neg. only \\
Real-World 2 & 30 & 83,817 & 20,955 & Lattices & 500 & neg. only \\
\hline
\end{tabular}

these restrictions then maps directly onto the Pipelined Set Cover problem [20], where the goal is to find the optimal ordering $\pi$ such that the ordered sequence of sets $S_{\pi(1)}(1), \ldots, S_{\pi(T)}(1)$ covers all possible training examples contained in $\cup_{t=1}^{T} S_{t}(1)$ while achieving the lowest total evaluation time cost.

Theorem 1 by Mungala et al. [20] shows that the greedy algorithm that chooses the set that minimizes the cost ratio each time achieves a 4-approximation of OPT. Since QWYC minimizes the evaluation time ratio $J_{r}$ when choosing the $r$ th base model and early stopping thresholds $\epsilon_{r}^{+}$ and $\epsilon_{r}^{-}$, QWYC achieves a 4-approximation of $O P T$.

Since in Pipeline, $O P T=O P T^{*}, \mathrm{QWYC}$ also achieves a 4-approximation of $O P T^{*}$ for problems in Pipeline.

Feige et al. [10] showed that problems in Pipeline with uniform cost do not admit to better than a 4-approximation unless $P=N P$.

\section{EXPERIMENTS}

Let QWYC* refer to Algorithm 1, which uses our proposed joint optimization of ordering and early stopping thresholds. To illustrate the effectiveness of joint optimization, we compare QWYC* to other ways to select the ordering, but still use the early stopping thresholds defined by our Algorithm 2. See Section 5.1 for details on the comparison ordering methods.

The closest method to QWYC* is arguably that of Fan et al. [9]: they select an ordering based on sorting the base models by their individual MSE, and they provide an early stopping mechanism that is more complex and flexible than Algorithm 2, which we in detail in Section 5.4. Let Fan* refer to using both their early stopping mechanism and their ordering by Individual MSE. We also compare their early stopping mechanism to that of Algorithm 2 for different methods of base model ordering.

We also compare to simply training a smaller ensemble in the first place, and then always fully evaluating it (no dynamic pruning). That may not be an option if one is handed a pre-trained ensemble and asked to speed it up, but it is an important comparison point for demonstrating the value of dynamic pruning in theory.

\subsection{Comparison Orderings}

We compare with the following methods that, like QWYC, determine one fixed ordering of the base models that is the same ordering for all examples. We pair each of the orderings with two different early stopping mechanisms to implement dynamic pruning: (i) the proposed QWYC early stopping mechanism of Algorithm 2, and (ii) the early stopping mechanism of Fan et al. [9].

Order by Individual MSE. As done by Fan et al. [9], one can choose the ordering of the base models $\pi$ based on each base model's individual accuracy or MSE, though this requires access to labeled examples. For other precision-recall trade-offs, the best individual ordering may be slightly different. 
Order by Greedy MSE. Ordering by individual MSE can cause many very accurate but highly correlated classifiers to all be ordered first. Theoretically, a more optimal strategy is to first choose the best individual model (by MSE), and then greedily choose each subsequent base model to minimize the MSE of the resulting subensemble $\sum_{t=1}^{r} f_{t}(x)$. This greedy ordering is similar to the gradient-boosted tree ordering and to other approaches used in non-dynamic ensemble pruning [19].

Gradient Boosted Tree (GBT) Ordering. GBT ensembles are trained in a greedily additive fashion, where each base regression tree $f_{t}$ is selected to be the one that most improves the training objective when added to the subensemble of size $t-1$. Thus, this training procedure produces a natural ordering of base trees that we compare to.

Random Ordering. Random ordering of the base models is a random permutation drawn uniformly from all possible permutations of $\{1, \ldots, T\}$. In each experiment, we report the mean and standard deviation of results across five random permutations.

\subsection{Constraint on Classification Churn}

To illustrate the tradeoff between fast evaluation and faithfulness to the full ensemble, we vary the $\alpha$ hyperparameter that controls the maximum \% classification differences from the full ensemble of experiments that use either the QWYC* joint optimization or Algorithm 2 with other pre-selected orderings. Similarly, for comparisons that use the Fan et al. [9] early-stopping mechanism, we vary their $\gamma$ hyperparameter that indirectly controls the $\%$ classification differences from the full ensemble.

\subsection{Datasets}

We demonstrate the performance of QWYC's joint optimization of ordering and early stopping thresholds on the four datasets detailed in Table 1: two benchmark datasets and two datasets from Google. For datasets without a pre-defined train/test split, we randomly shuffle the dataset and split it 80-20 to produce a train and test set. The train set is used to optimize the ordering and early stopping thresholds.

\subsection{Details on Early Stopping Criteria by Fan et al. [9]}

We compare the performance of $\mathrm{QWYC}^{*}$ to that of the dynamic ensemble pruning strategy proposed by Fan et al. that they refer to as dynamic scheduling [9]. Fan et al. recommend that the base models be ordered by decreasing total benefits, where total benefits may be application specific, but as a default implementation they suggest using the mean squared error (MSE) of the individual base model. We refer to this ordering as Individual MSE. In preliminary experiments, we found ordering by the models' individual MSE's to be more useful than ordering by their training accuracies.

For a given order of the base models, Fan et al. [9] choose multiple thresholds per base model, one per bin of incomplete scores. For clarity, we describe our implementation of this in some detail. They originally apply their method to ensembles where the base models are averaged, but here we straightforwardly extend their method to ensembles where the base models are summed. Let $g_{r}(x)=\sum_{t=1}^{r} f_{t}(x)$ denote the partial ensemble evaluation up through base model $r$.

For each $r$, define a distinct set $\mathcal{B}_{r}$ of bins into which $g_{r}(x)$ will be mapped, and let $b_{r}(x) \in \mathcal{B}$ denote the bin that $g_{r}(x)$ is assigned to.

For every bin $B_{r} \in \mathcal{B}_{r}$, let $\mu_{B_{r}}, \sigma_{B_{r}}^{2}$ denote the empirical mean and variance of the differences compared to the full ensemble evaluation for a set of representative examples that are mapped to $B_{r}$. Let $\gamma$ be a confidence hyperparameter that is set by the user to control the accuracy of the early stopping with respect to the full ensemble evaluation. Then, the early stopping thresholds after 
the $r$ th base model evaluation and the bin $B_{r}$ are:

$$
\epsilon_{r, B_{r}}^{+}=\mu_{B_{r}}+\gamma \sigma_{B_{r}} \quad \text { and } \quad \epsilon_{r, B_{r}}^{-}=\mu_{B_{r}}-\gamma \sigma_{B_{r}} .
$$

Fan et al. [9] does not specify a concrete method for how to map $g_{r}(x)$ to a bin. We found the best and most efficient results using rounding and a knob $\lambda$ to control how many bins there are: bin $g_{r}(x)$ as $b_{r}(x)=$ floor $\left(g_{r}(x) / \lambda\right)$. As recommended by Fan et al., we map the bin values to confidence parameters using a hash table to save evaluation time. For all experiments, we vary the knob $\lambda$ between 0.1 and 0.001 to test more or fewer bins, causing the mean number of bins per base model to range from 10 to 400 . If one uses a large number of bins (small $\lambda$ ), we found too few training examples fell into each bin and led to high generalization error, and that a value of $\lambda=0.01$ produced the best tradeoff between speed and accuracy for most experiments.

Recall the full model $f(x)$ is thresholded at $\beta$ to form a classifier. Then the decision rule for each example $x$ after the $r$ th base model evaluation is:

$$
\begin{cases}g_{r}(x)>\beta+\epsilon_{r, b_{r}(x)}^{+}, & \text {classify positive } \\ g_{r}(x)<\beta+\epsilon_{r, b_{r}(x)}^{-}, & \text {classify negative } \\ \text { otherwise } & \text { continue evaluating. }\end{cases}
$$

It can happen that during evaluation time, an example $x$ is mapped to a bin that does not exist in the hash table if no examples were mapped to that bin on a train set. In that case, Fan et al. [9] say to perform the full evaluation for $x$, which is what we do in such cases. In our experiments, this happened only for around 10 examples.

\subsection{Benchmark Experiments Set-Up}

For the Adult and the Nomao benchmark datasets from the UCI Machine Learning Repository [8], we train gradient boosted tree (GBT) ensemble models [12]. A GBT ensemble model is an additive model where the output is the sum of $T$ regression trees. The training process produces a natural ordering of the trees because each tree is added to the ensemble sequentially, and we compare this ordering to QWYC's optimized ordering in experiments. When training the full ensemble, we perform hyperparameter optimization on a validation set over the number of base trees in the ensemble, the maximum depth of a single base tree, and the learning rate. We treat the evaluation cost of each base model as a constant $c_{t}=1$ for all $t$, which is a conservative assumption as the maximum depth of each base tree is bounded.

We compare the performance of QWYC* to the Fan et al. [9] early stopping method with their suggested pre-selected ordering by Individual MSE. We also compare QWYC* to a baseline of training smaller GBT ensembles of varied sizes without pruning. Finally, since GBTs produce a natural ordering of base models in the training process, we compare to the additional baseline of using the original GBT ordering as a pre-selected ordering.

For these two datasets, Figure 1 shows the tradeoff between $\%$ test accuracy and mean \# base models, and Figure 2 shows the tradeoff between $\%$ classification differences on the test set and mean \# base models. See Section 6 for a discussion of the results.

Experiment 1: UCI Adult Dataset. This is a binary classification task where the objective is to predict whether or not a person's income is greater than $\$ 50,000$. The full ensemble has $T=500$ base trees of maximum depth 5 and acts on a total of $D=14$ features. We use the predefined train/test split provided in the repository.

Experiment 2: UCI Nomao Dataset. This is a binary classification task where the goal is entity resolution: each labeled example contains information about two sources, and the label is whether 


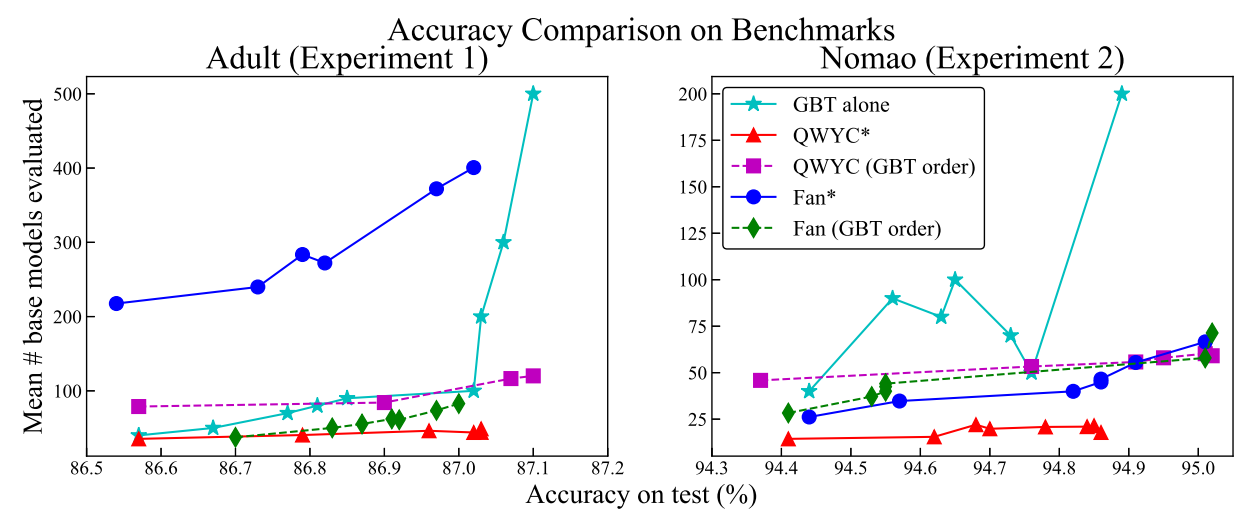

Fig. 1. Test accuracy vs. mean \# base models evaluated for Adult and Nomao experiments. QWYC (GBT order) refers to Algorithm 2 with pre-selected GBT ordering, and Fan (GBT order) refers to Fan et al. [9] early stopping mechanism with pre-selected GBT ordering.

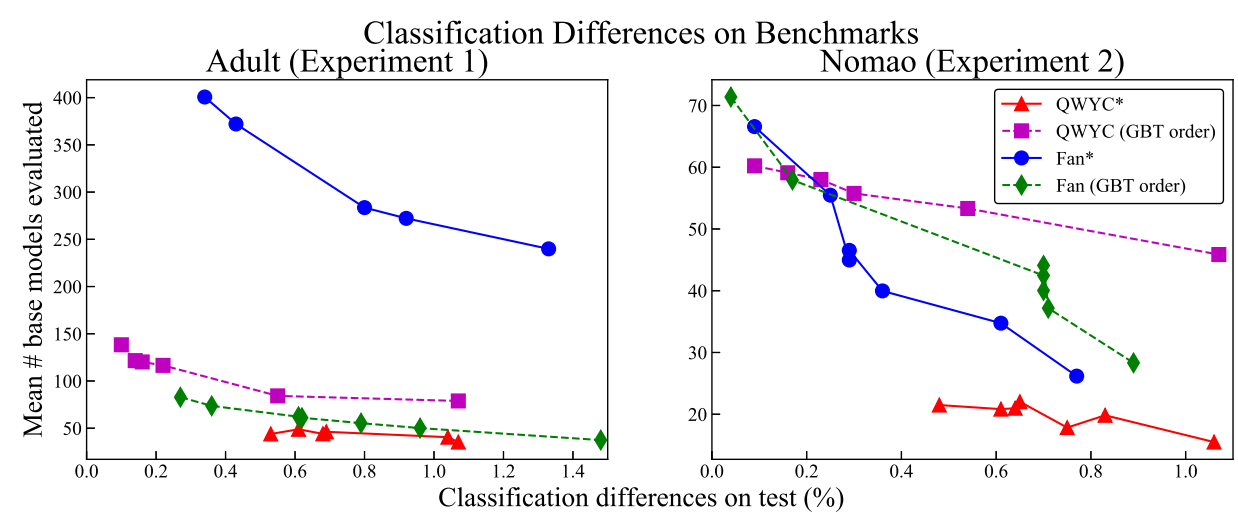

Fig. 2. Test classification differences vs. mean \# base models evaluated for Adult and Nomao experiments. The classification differences on test (\%) is given by $\frac{\text { \# classification differences w.r.t. full ensemble }}{\text { total \# of test examples }} * 100$.

those two sources refer to the same business. The full ensemble has $T=500$ base trees of $\max$ depth 9 and uses the strongest $D=8$ out of 120 available features.

\subsection{Real-World Experiments Set-Up}

We experiment with two proprietary real-world filter-and-score problems from Google, where the goal is to quickly filter out the negative examples, and if the algorithm makes a positive classification then the full ensemble score $f(x)$ is required for further processing by later stages. Thus, only $\epsilon^{-}$is optimized, and evaluation cost can only be reduced on negative examples.

These problems are examples where fast average processing and small total CPU usage are very desirable. For example, in practice the real-world problem 1 requires evaluating the model over one trillion times a day, and each evaluation is latency-sensitive because it is in response to a real-time request.

For these real-world experiments, we were given ensemble models where each base model is a two-layer calibrated lattice model [6]. Lattices are interpolated look-up tables, and can be trained with the open-source package TensorFlow Lattice. Lattices are similar in evaluation time and flexibility to decision trees, but have the advantages that they enable monotonicity constraints and 
produce smooth, continuous real-valued outputs $f_{t}(x) \in \mathbb{R}[6]$, rather than the piecewise-constant outputs of standard decision trees. The evaluation time for each lattice base model will be roughly equal, since each lattice acts on the same number of features, so we model the base model evaluation costs as $c_{t}=1$ for all $t$. We were given jointly trained ensembles, but we retrained each ensemble with independent-training of the base models to be able to compare speed-ups for independently trained vs. jointly trained.

Figure 3 shows the tradeoff between $\%$ classification differences and mean \# base models evaluated for Experiment 3. For these datasets, the held-out test examples are not all labeled, so we do not report test accuracies for these experiments.

For these experiments, we also report timing numbers for evaluating QWYC* and Fan* with proprietary highly optimized evaluation code, constrained for $\approx 0.5 \%$ classification differences in Tables 2 and 3. This amount of allowed difference was chosen based on a rough estimate of the percent of examples for which the ground truth label is likely noisy. We report the mean and standard deviation of the per-example evaluation time on the test set after 100 runs.

Similarly, for the independently trained ensembles of Experiments 5-6, Figure 4 shows the tradeoff between \% classification differences and mean \# base models evaluated on the independently trained ensembles. We report timings for roughly $0.5 \%$ test set classification differences for Experiments 5-6 in Tables 4 and 5.

Results are discussed in Section 6.

Note that for all of the real-world filter-and-score Experiments 3-6, we only allow for early stopping on examples that the early stopping mechanism determines to be a negative classification and always evaluate the full ensemble on positive examples. Therefore, since the class priors are roughly equal in Experiments 4 and 6, the maximum possible speedup factor under the $0.5 \%$ classification difference constraint is approximately $2 \times$.

Experiment 3: Real-World 1, fointly Trained. For this real-world filter-and-score problem, the full ensemble contains $T=5$ base models, and the base models were jointly trained. Each of the $T=5$ base models acts on 13 features out of a total set of $D=16$ features, where the feature subsets for the 5 base models are chosen to maximize the interactions of the features in each base model as per the crystals ensemble-construction method of Canini et al. [6]. On the one hand, this is a particularly challenging ensemble to speed up because there are so few base models and the base models were jointly trained to work together to maximize accuracy, but on the other hand, each base model has a rich feature representation, so the hope is that easy examples can be decided with just one or two base models, and that the full ensemble may only be needed for hard examples. The train dataset has 183, 755 examples and the test dataset has 45, 940. The task is heavily biased to rejecting candidates: a priori, the probability a sample will be classified negative by the full ensemble is 0.95 .

Experiment 4: Real-World 2, fointly Trained. For our second real-world filter-and-score problem, the full ensemble contains $T=500$ base models, and the base models were all jointly trained. Each base model acted on eight features out of a total set of $D=30$ features, and the feature subset for each of the 500 base models was jointly optimized across all base models according the methodology outlined in Section 3.1 of Canini et al. [6], which means that some base models can be expected to be much more powerful than others. The ensemble was jointly trained, but still we expect not all $T=500$ lattices will be needed for every example's decision, so this is a compelling case for dynamic pruning. The train dataset has 83,817 examples and the test dataset has 20,955. The class priors are roughly equal.

Experiments 5 and 6: Real-World Problems 1 and 2, Independently Trained. Experiments 5 and 6 use the same data and set-up as Experiments 3 and 4 respectively, except the base models are independently trained instead of jointly trained. For the independently trained full ensemble, each 


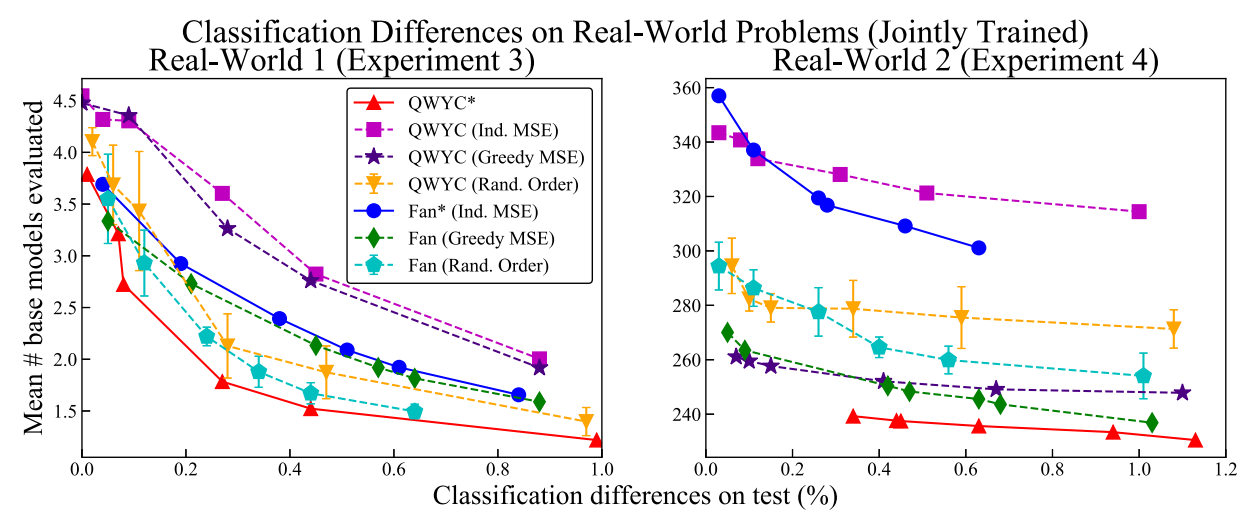

Fig. 3. Classification differences vs. mean \# base models evaluated for real-world filter-and-score experiments with jointly trained ensembles. The error bars for Random Order show the standard deviation after 5 trials with different random orderings.

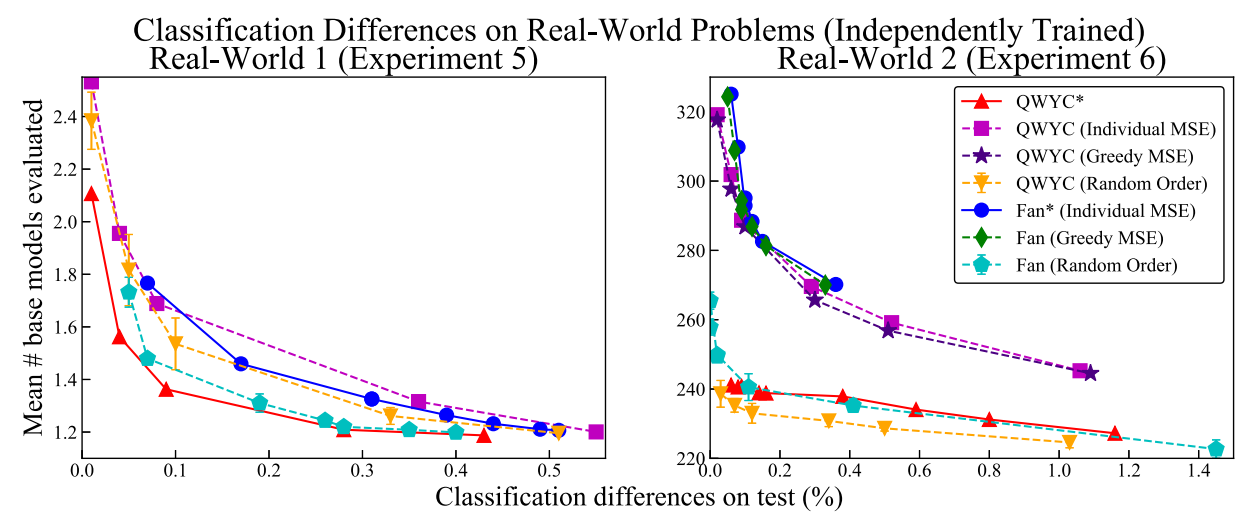

Fig. 4. Classification differences vs. mean \# base models evaluated for real-world experiments with independently trained ensembles.

Table 2. Experiment 3: Comparison of Evaluation Times for Jointly Trained Ensemble of Five Base Models on the Test Set

\begin{tabular}{lcccc}
\hline & $\begin{array}{c}\text { \% differences } \\
\text { vs. full ensemble }\end{array}$ & $\begin{array}{c}\text { Mean \# base } \\
\text { models evaluated }\end{array}$ & $\begin{array}{c}\text { Mean evaluation } \\
\text { time }(\mu \mathrm{s})\end{array}$ & Mean speed-up \\
\hline Full ensemble & 0 & 5 & $4.98 \pm 6 \%$ & $1 \times$ \\
QWYC $^{*}$ & $0.44 \%$ & 1.54 & $1.82 \pm 7 \%$ & $2.7 \times$ \\
Fan $^{*}$ & $0.51 \%$ & 2.09 & $2.49 \pm 8 \%$ & $2.0 \times$ \\
\hline
\end{tabular}

base model's subset of eight features was randomly selected with replacement from the total set of features.

\section{DISCUSSION OF EXPERIMENTAL RESULTS}

Overall, QWYC* works robustly and effectively. In Experiments 1-5, QWYC*'s joint optimization of ordering and early stopping thresholds outperformed any of the other fixed orderings combined with Algorithm 2, and the early stopping strategy of Fan et al. [9], in terms of tradeoffs between \# 
Table 3. Experiment 4: Comparison of Evaluation Times for Jointly Trained Ensemble of 500 Base Models on the Test Set

\begin{tabular}{lcccc}
\hline & $\begin{array}{c}\text { \% differences } \\
\text { vs. full ensemble }\end{array}$ & $\begin{array}{c}\text { Mean \# base } \\
\text { models evaluated }\end{array}$ & $\begin{array}{c}\text { Mean evaluation } \\
\text { time }(\mu \mathrm{s})\end{array}$ & Mean speed-up \\
\hline Full ensemble & 0 & 500 & $322 \pm 4 \%$ & $1 \times$ \\
QWYC $^{*}$ & $0.45 \%$ & 237 & $177 \pm 4 \%$ & $1.8 \times$ \\
Fan $^{*}$ & $0.46 \%$ & 309 & $293 \pm 12 \%$ & $1.1 \times$ \\
\hline
\end{tabular}

Table 4. Experiment 5: Comparison of Evaluation Times for Independently Trained Ensemble of Five Base Models on the Test Set

\begin{tabular}{lcccc}
\hline & $\begin{array}{c}\text { \% differences } \\
\text { vs. full ensemble }\end{array}$ & $\begin{array}{c}\text { Mean \# base } \\
\text { models evaluated }\end{array}$ & $\begin{array}{c}\text { Mean evaluation } \\
\text { time }(\mu \mathrm{s})\end{array}$ & Mean speed-up \\
\hline Full ensemble & 0 & 5 & $4.91 \pm 7 \%$ & $1 \times$ \\
QWYC $^{*}$ & $0.43 \%$ & 1.18 & $1.23 \pm 6 \%$ & $4.0 \times$ \\
Fan* $^{*}$ & $0.51 \%$ & 1.20 & $1.39 \pm 7 \%$ & $3.5 \times$ \\
\hline
\end{tabular}

Table 5. Experiment 6: Comparison of Evaluation Times for Independently Trained Ensemble of 500 Base Models on the Test Set

\begin{tabular}{lcccc}
\hline & $\begin{array}{c}\text { \% differences } \\
\text { vs. full ensemble }\end{array}$ & $\begin{array}{c}\text { Mean \# base } \\
\text { models evaluated }\end{array}$ & $\begin{array}{c}\text { Mean evaluation } \\
\text { time }(\mu \mathrm{s})\end{array}$ & Mean speed-up \\
\hline Full ensemble & 0 & 500 & $269 \pm 4 \%$ & $1 \times$ \\
QWYC $^{*}$ & $0.59 \%$ & 234 & $149 \pm 4 \%$ & $1.8 \times$ \\
Fan* $^{*}$ & $0.46 \%$ & 268 & $209 \pm 7 \%$ & $1.3 \times$ \\
\hline
\end{tabular}

base models evaluated and \% classification differences and accuracy (see Figures 1, 2, 3, and 4). For Experiment 6, as shown in Figure 4, QWYC* was tied for second best ordering, and actually the best results were with a random ordering-Experiment 6 used 500 base models and the ensemble was independently trained, so many of the 500 base models may have behaved similarly, and we believe this noisy setting caused the optimized orderings to simply overfit. Experiment 4 was with the same dataset and same 500 base models but jointly trained, and Figure 3 shows in that setting that random ordering performs much worse than QWYC*.

\subsection{Dynamic Pruning vs. Smaller Ensemble}

The experiments also illustrate the practical benefits of dynamic ensemble pruning over simply training with a smaller fixed number of base models. Specifically, see the line marked GBT alone in Figure 1, which shows the relationship between the number of base models used in the gradient boosted tree ensemble and its full unpruned evaluation accuracy on the x-axis. For every achievable accuracy, QWYC* requires evaluating fewer base models on average compared to training a smaller ensemble. This is also true if the Fan early stopping mechanism is used with the full GBT ensemble's original GBT ordering, marked as Fan (GBT order). However, a surprising result was that in Experiment 1 with the GBT ensemble, Fan* performed worse than fully evaluating a fixed-size smaller ensemble, both in accuracy and classification differences. This appears to happen because Fan* used Individual MSE ordering, which is prone to ignoring diversity in base models, since using the Fan early stopping with the GBT order (marked Fan (GBT order)) did perform better than fully evaluating a smaller ensemble. 


\subsection{Timing Results}

The above results show that QWYC* allows us to evaluate fewer base models on average than other methods to achieve a given accuracy. Tables 2-5 also show that mean evaluation times are faster with QWYC*. For example, QWYC* produced a $2.7 \times$ speed-up for the jointly-trained ensemble of $T=5$ base models in Table 2, which is evaluated trillions of times each day. Because the base models (lattices) in these ensembles are all roughly computationally equal to evaluate, these timing numbers are also reflective of total CPU savings.

Larger gains were shown in the dynamic ensemble pruning study of Basilico et al. [1], but those experiments used very large independently trained ensembles (up to 1 million independently trained base models), which have significantly more headroom to speed up. In contrast, our experiments include much smaller jointly trained ensembles, which are less likely to contain redundant base models. Even in our experiments on independently trained ensembles (Experiments 4 and 6), due to the previous class and the filter-and-score modality that only enables early decisions for negative examples, the maximum possible speed-up is $2 \times$, and QWYC achieves close to this bound with a $1.8 \times$ speed-up, both for the jointly trained and independently-trained ensembles, as reported in Tables 3 and 5.

\subsection{Comparison of Early Stopping Mechanisms}

For many of the different orderings and experiments, the Fan et al. [9] early stopping mechanism actually performed better than the proposed QWYC early stopping mechanism given in Algorithm 2. Note that the Fan et al. [9] early stopping mechanism is significantly more flexible than Algorithm 2, with at least 20 thresholds per base model (2 thresholds per bin) rather than just 2 early-stopping thresholds per base model in Algorithm 2. This suggests that the QWYC base model ordering could potentially achieve an even greater reduction in the mean number of base models evaluated if combined with this more flexible early stopping mechanism Fan et al. [9], but the increased complexity of the early stopping of Fan et al. [9] takes more evaluation time as the number of early stopping bins is increased. Given the increased complexity of the Fan early stopping mechanism, it is an open question of how to effectively jointly optimize the QWYC ordering and the Fan early stopping parameters.

\subsection{Performance on Jointly vs. Independently Trained Ensembles}

For both real-world filter-and-score problems, we see better speedups for independently trained ensembles than for jointly trained ensembles. This is likely because when then ensemble is independently trained, each base model correlates more strongly with the full evaluation than when the ensemble is jointly trained and base models can specialize. This effect is most pronounced for Experiment 1, with an average $4 \times$ speed-up for the independently trained ensemble (Table 4) compared to a $2.7 \times$ speedup for the jointly-trained ensemble (Table 2). The difference is much smaller for Experiment 2, but the mean number of base models evaluated is slightly lower for QWYC in Table 5.

For independently trained ensembles, the ordering mattered less, and a random ordering sometimes produced even better test set speed-ups than the orderings optimized on the train set; a failure of those optimized orderings to generalize well. In Experiment 6, Figure 4, the random orderings performed significantly better than both Greedy MSE and Individual MSE orderings. Even in cases where the more clever Greedy MSE and Individual MSE orderings failed, QWYC's joint optimization of ordering and thresholds in Algorithm 1 was able to replicate at least statistically similar performance to the best random ordering. 


\section{CONCLUSIONS AND EXTENSIONS}

Our main contribution is to demonstrate both the value and practicality of jointly optimizing the ordering and early stopping mechanism for dynamic ensemble pruning, and the gains possible on average time and CPU needed to evaluate each example. We give an algorithm QWYC* that approximately solves the combinatorial joint optimization, achieving the best approximation bound in polynomial time for certain cases. Experimentally, we focused on the case of ensembles that have already been well optimized for size, in that they are sequentially or jointly trained, and the total number of base models has already been optimized for the full ensemble. In this setting, we demonstrated that QWYC ${ }^{*}$ s joint optimization produces the fastest early classifier with a constraint on how faithful it is to the full evaluation.

Our comparisons of early stopping mechanisms show that the more complex early stopping of Fan et al. [9] can be more efficient than the simpler early stopping that we used for QWYC*, and an open question is how to jointly optimize the QWYC ordering with this more flexible early stopping mechanism, and whether the actual evaluation time would be faster, or whether gains would be swallowed in the slower per-model evaluation time. Further, there may be other early stopping mechanisms that might work more efficiently or be easier to optimize with the ordering.

\subsection{Finding a Better Optimum}

The QWYC optimization algorithm presented here does not guarantee a global optimum of the proposed objective. It is theoretically possible to improve the optimization further by jointly optimizing the orderings at multiple evaluation stages. For example, one could optimize $\pi(1)$ and $\pi(2)$ jointly, then $\pi(2)$ and $\pi(3)$ jointly, and proceed iteratively from there, keeping the same method of $\epsilon$ optimization. If $n$ is the number of ordering stages to optimize jointly, then the complexity of jointly optimizing $n$ stages is $O\left(T^{n+1} N\right)$. The extra complexity might be worthwhile for very latency sensitive applications, but it is an open question how much could be gained by improving the optimization.

\subsection{Extensions beyond Binary Classification}

While we focused here on binary classification, it is straightforward to extend the proposed optimization strategy to multi-class classifiers. Whether a similar dynamic pruning strategy can be effectively devised for ranking or regression is an open question. This section discusses these additional use cases and possible extensions to the QWYC objective function (3).

7.2.1 Multi-Class Classifiers. It is straightforward to generalize (3) to the multi-class case in which there are $K$ classes. Replace (1) by $K$ ensembles, each providing a score in favor of the $k$ th class:

$$
f^{k}(x)=\sum_{t=1}^{T} f_{t}^{k}(x) \text { for } k=1, \ldots, K .
$$

We then solve an extended version of the optimization problem in Equation (3) for $K$ threshold vectors $\left\{\epsilon^{k} \in \mathbb{R}^{T}\right.$ for $\left.k=1, \ldots, K\right\}$ to control the early decisions for each of the classes.

Let $g_{r}^{k}(x, \pi)=\sum_{t=1}^{r} f_{\pi(t)}^{k}(x)$. After evaluating the $r$ th base model for $r \in\{1, \ldots, T\}$, an example $x$ belongs to one of $K+1$ mutually exclusive sets: the sets where $x$ is predicted to belong to class $k, P_{r}^{k}$ for $k=1, \ldots, K$, and the uncertain set $U_{r}:\left\{x: x \notin \bigcup_{k=1}^{K} P_{r}^{k}\right\}$. Defining mutually exclusive sets $P_{r}^{k}$ has an additional layer of complexity in the multi-class setting, but it is still possible. For example, one could define $P_{r}^{k}:=\left\{x \mid g_{r}^{k}(x, \pi)>\epsilon_{r}^{k}\right.$ and $g_{r}^{j}(x, \pi)<\epsilon_{r}^{j}$ for all $\left.j \neq k\right\}$. If $x \in P_{r}^{k}$ then the fast classifier classifies $x$ as the $k$ th class and we terminate the evaluation. Otherwise, 
for $x \in U_{r}$, the classification decision is not yet uncertain, and we continue on to evaluate the $\pi(r+1)$ th base model for each of the $K$ classes. Let $C_{r}:=\bigcap_{t=0}^{r} U_{t}$ denote the set of samples that remain unclassified after evaluating the $r$ th base model.

For the full classifier, let $P_{\text {full }}^{k}$ represent the set of examples predicted by the full ensemble to belong to class $k$, for $k=1, \ldots, K$. Let $N_{\text {full }}^{k}=\bigcup_{j \neq k} P_{\text {full }}^{j}$ represent the set of examples predicted by the full ensemble to not belong to class $k$.

The extended version of Equation (3) is given by

$$
\begin{aligned}
\underset{\pi \in \Pi, \epsilon^{1}, \ldots, \epsilon^{K} \in \mathbb{R}^{T}}{\operatorname{minimize}} & \frac{1}{N} \sum_{t=1}^{T} \sum_{i=1}^{N} c_{\pi(t)} \mathbf{1}\left[x_{i} \in C_{t-1}\right] \\
\text { subject to } & h_{T}\left(\epsilon^{1}, \ldots, \epsilon^{K}, \pi\right) \leq \alpha,
\end{aligned}
$$

where $h_{T}\left(\epsilon^{1}, \ldots, \epsilon^{K}, \pi\right)$ represents the fraction of classification differences between the fast classifier and the full classifier:

$$
h_{T}\left(\epsilon^{1}, \ldots \epsilon^{K}, \pi\right)=\frac{1}{N} \sum_{i=1}^{N} \sum_{k=1}^{K} 1\left[x_{i} \in \bigcup_{t=1}^{T}\left\{C_{t-1} \cap P_{t}^{k} \cap N_{\text {full }}^{k}\right\}\right] .
$$

7.2.2 Precision-Recall Variant. A variant of this problem is to separately constrain the false positive (FP) and false negative (FN) rates compared to the full evaluation, controlling them with separate hyperparameters $\alpha_{F P}$ and $\alpha_{F N}$ :

$$
\begin{array}{ll}
\underset{\pi \in \Pi, \epsilon^{+} \in \mathbb{R}^{T}, \epsilon^{-} \in \mathbb{R}^{T}}{\operatorname{minimize}} & \mathbb{E}_{X}\left[\sum_{t=1}^{T} c_{\pi(t)} I_{X_{i}, t, \pi, \epsilon^{+}, \epsilon^{-}}\right] \\
\text {subject to } & \mathbb{E}_{X}\left[F P_{X, t, \pi, \epsilon^{+}}\right] \leq \alpha_{F P}, \\
& \mathbb{E}_{X}\left[F N_{X, t, \pi, \epsilon^{-}}\right] \leq \alpha_{F N}, \\
& \epsilon^{-} \leq_{+} \epsilon^{+},
\end{array}
$$

where $I_{X_{i}, t, \pi, \epsilon^{+}, \epsilon^{-}}$is a Bernoulli random variable that is 1 if $X$ is classified after $t$ base models, and 0 otherwise, and $F P$ and $F N$ are Bernoulli random variables indicating whether the incomplete classification of $X$ caused a false positive or false negative compared to the complete classifier. Similar to Equation (3), it is straightforward to express an empirical analogue for Equation (7).

7.2.3 Ranking. If the goal of evaluating $f(x)$ is not to classify $x$, but rather to evaluate a set of candidates $\left\{x_{j}\right\}$ and rank all of them, it may similarly be possible to evaluate only some subset of the $T$ base models for each $x_{j}$ and still provide almost-as good ranking of all the set $\left\{x_{j}\right\}$ by sorting the fast scores, since noisy scores may still provide the same ranking. But it is less clear how to frame good criteria for selecting the base model permutation and early stopping criterion, and we leave this case as an open question.

\section{REFERENCES}

[1] J. D. Basilico, M. A. Munson, T. G. Kolda, K. R. Dixon, and W. P. Kegelmeyer. 2011. COMET: A recipe for learning and using large ensembles on massive data. In IEEE International Conference on Data Mining (ICDM).

[2] D. Benbouzid, R. Busa-Fekete, and B. Kégl. 2012. Fast classification using sparse decision DAGs. In International Conference on Machine Learning (ICML).

[3] L. Bourdev and J. Brandt. 2005. Robust object detection via soft cascade. In Conference on Computer Vision and Pattern Recognition (CVPR).

[4] L. Breiman. 2001. Random forests. Machine Learning 45, 1 (2001), 5-32.

[5] R. L. Burden and J. D. Faires. 1985. Numerical Analysis (3rd ed.). PWS Publishers. 
[6] K. Canini, A. Cotter, M. M. Fard, M. R. Gupta, and J. Pfeifer. 2016. Fast and flexible monotonic functions with ensembles of lattices. In Advances in Neural Information Processing Systems (NIPS).

[7] R. M. O. Cruz, R. Sabourin, and George D. C. Cavalcanti. 2015. META-DES.H: A dynamic ensemble selection technique using meta-learning and a dynamic weighting approach. International foint Conference on Neural Networks (IFCNN) (2015).

[8] Dheeru Dua and Casey Graff. 2017. UCI Machine Learning Repository. http://archive.ics.uci.edu/ml.

[9] W. Fan, F. Chu, H. Wang, and P. S. Yu. 2002. Pruning and dynamic scheduling of cost-sensitive ensembles. In $A A A I$ Conference on Artificial Intelligence.

[10] U. Feige, L. Lovasz, and P. Tetali. 2004. Approximating min-sum set cover. Algorithmica (2004).

[11] M. Fernandez-Delgado, E. Cernadas, S. Barro, and D. Amorim. 2014. Do we need hundreds of classifiers to solve real world classification problems? Journal of Machine Learning Research ( $7 M L R)$ (2014).

[12] J. Friedman. 2001. Greedy function approximation: A gradient boosting machine. Annals of Statistics 29 (2001), 11891232. Issue 5.

[13] T. Gao and D. Koller. 2011. Active classification based on value of classifier. In Advances in Neural Information Processing Systems (NIPS).

[14] T. Hastie and R. Tibshirani. 1990. Generalized Additive Models. Chapman Hall, New York.

[15] D. Hernandez-Lobato, G. Martinez-Munoz, and A. Suarez. 2009. Statistical instance-based pruning in ensembles of independent classifiers. IEEE Transactions on Pattern Analysis and Machine Intelligence (TPAMI) 31, 2 (2009), 364-369.

[16] T. Kim, I. Budvytis, and R. Cipolla. 2012. Making a shallow network deep: Conversion of a boosting classifier into a decision tree by Boolean optimisation. International fournal of Computer Vision (2012).

[17] S. Z. Li, Z. Q. Zhang, H. Shum, and H. J. Zhang. 2002. FloatBoost learning for classification. In Advances in Neural Information Processing Systems (NIPS).

[18] D. Margineantu and T. Dietterich. 1997. Pruning adaptive boosting. In International Conference on Machine Learning (ICML).

[19] G. Martinez-Munoz and A. Suarez. 2006. Pruning in ordered bagging ensembles. In International Conference on Machine Learning (ICML).

[20] K. Munagala, S. Babu, R. Motwani, and J. Widom. 2005. The pipelined set cover problem. In International Conference on Database Theory.

[21] F. Nan, J. Wang, and V. Saligrama. 2016. Pruning random forests for prediction on a budget. In Advances in Neural Information Processing Systems (NIPS).

[22] A. Niculescu-Mizil, C. Perlich, G. Swirszcz, V. Sindhwani, Y. Liu, P. Melville, D. Wang, J. Xiao, J. Hu, M. Singh amd W. X. Shang, and Y. F. Zhu. 2009. Winning the KDD cup orange challenge with ensemble selection. In International Conference on KDD-Cup, Vol. 7. JMLR.org, 23-34.

[23] N. Parrish, H. Anderson, M. R. Gupta, and D. Y. Hsaio. 2013. Classifying with confidence from incomplete information. Journal of Machine Learning Research (FMLR) 14 (2013), 3561-3589.

[24] C. Qian, Y. Yu, and Z. H. Zhou. 2015. Pareto ensemble pruning. In AAAI Conference on Artificial Intelligence.

[25] M. J. Saberian and N. Vasconcelos. 2010. Boosting classfier cascades. In Advances in Neural Information Processing Systems (NIPS).

[26] A. Santana, R. G. F. Soares, A. M. P. Canuto, and M. C. P. Souto. 2006. A dynamic classifier selection method to build ensembles using accuracy and diversity. In Brazilian Symposium on Neural Networks (SBRN). 36-41.

[27] E. M. D. Santos, R. Sabourin, and P. Maupin. 2007. Ambiguity-guided dynamic selection of ensemble of classifiers. In International Conference on Information Fusion.

[28] G. A. Schwing, C. Zach, Y. Zheng, and M. Pollefeys. 2011. Adaptive random forest - How many experts to ask before making a decision? In Conference on Computer Vision and Pattern Recognition (CVPR).

[29] J. Sochman and J. Matas. 2005. Waldboost-learning for time constrained sequential detection. In Conference on Computer Vision and Pattern Recognition (CVPR).

[30] V. Soto, A. Suarez, and G. Martinez-Munoz. 2016. An urn model for majority voting in classification ensembles. In Advances in Neural Information Processing Systems (NIPS).

[31] P. Sun and J. Zhou. 2013. Saving evaluation time for the decision function in boosting: Representation and reording base learner. In International Conference on Machine Learning (ICML).

[32] C. Tamon and J. Xiang. 2000. On the boosting pruning problem. In European Conference on Machine Learning (ECML).

[33] A. Verikas, A. Lipnickas, K. Malmqvist, Bacauskiene, and Adas Gelzinis. 1999. Soft combination of neural classiers: A comparative study. Pattern Recognition Letters 20, 4 (1999), 429-444.

[34] P. Viola and M. Jones. 2001. Robust real-time object detection. In ICCV Workshop on Statistical and Computational Theories of Vision.

[35] J. Wang, K. Trapeznikov, and V. Saligrama. 2015. Efficient Learning by directed acyclic graph for resource constrained prediction. In Advances in Neural Information Processing Systems (NIPS). 
[36] D. H. Wolpert. 1992. Stacked generalization. Neural Networks 5 (1992).

[37] K. Woods, W. P. Kegelmeyer, and K. Bowyer. 1997. Combination of multiple classifiers using local accuracy estimates. IEEE Transactions on Pattern Analysis and Machine Intelligence 19 (1997), 405-410. Issue 4.

[38] J. Xiao, C. He, X. Jiang, and D. Liu. 2010. A dynamic classifier ensemble selection approach for noise data. Information Sciences 180 (2010), 3402-3421. Issue 18.

[39] Z. Xu, M. J. Kusner, K. Q. Weinberger, and M. Chen. 2013. Cost-sensitive tree of classifiers. In International Conference on Machine Learning (ICML).

[40] C. Zhang and P. Viola. 2007. Multiple-instance pruning for learning efficient cascade detectors. In Advances in Neural Information Processing Systems (NIPS).

[41] S. Zhou. 2005. A binary decision tree implementation of a boosted strong classifier. In IEEE Workshop on analysis and modeling of faces and gestures.

[42] Z. H. Zhou, J. Wu, and W. Tang. 2002. Ensembling neural networks: many could be better than all. Artificial Intelligence 137 (2002), 239-263. Issue 1-2.

Received April 2020; revised December 2020; accepted February 2021 